\title{
A lentivirus-mediated miR-23b sponge diminishes the malignant phenotype of glioma cells in vitro and in vivo
}

\author{
LUYUE CHEN, KAILIANG ZHANG, ZHENDONG SHI, ANLING ZHANG, \\ ZHIFAN JIA, GUANGXIU WANG, PEIYU PU, CHUNSHENG KANG and LEI HAN
}

\begin{abstract}
Department of Neurosurgery, Tianjin Medical University General Hospital; Tianjin Neurological Institute; Key Laboratory of Post-Trauma Neuro-Repair and Regeneration in the Central Nervous System, Ministry of Education; Tianjin Key Laboratory of Injuries, Variations and Regeneration of the Nervous System, Tianjin 300052, P.R. China
\end{abstract}

Received September 27, 2013; Accepted October 21, 2013

DOI: $10.3892 /$ or.2014.3012

\begin{abstract}
RNA (miRNA) sponges are RNA molecules with repeated miRNA binding sequences that can sequester miRNAs from their endogenous target mRNAs, and a stably expressed miRNA sponge is particularly valuable for longterm loss-of-function studies in vitro and in vivo. Glioblastoma multiforme (GBM) is the most common primary brain tumor in adults and is characterized by extraordinarily angiogenic, invasive and migratory capabilities, hallmark features that make the disease incurable. Nonetheless, improvements in clinical treatment and a better understanding of the underlying molecular mechanisms have been achieved within the past few decades. miR-23b has previously been found to function as a tumor oncogene in GBM. In the present study, we employed an microRNA sponge that was forcibly expressed using a lentiviral vector to knock down the expression of miR-23b in vitro and in vivo and assessed the pleiotropic effects on glioma angiogenesis, invasion and migration. We demonstrated that the inhibition of miR-23b in glioma cell lines and orthotopic tumor mouse models resulted in a reduction in tumor malignancy, through the downregulation of HIF-1 $\alpha$, $\beta$-catenin, MMP2, MMP9, VEGF and ZEB1 and increased expression of VHL and E-cadherin. Therefore, we suggest that this miR-23b sponge could be developed into a promising anticancer therapy either alone or in combination with current targeted therapies.
\end{abstract}

\section{Introduction}

Currently, knockout animal models, microRNA antisense inhibitor oligonucleotides (1-3) and miRNA sponges (4)

Correspondence to: Professor Chunsheng Kang or Dr Lei Han, Laboratory of Neuro-Oncology, Tianjin Neurological Institute, Tianjin Medical University General Hospital, Tianjin 300052, P.R. China

E-mail: kang97061@gmail.com

E-mail: superhanlei@hotmail.com

Key words: malignant phenotype, microRNA sponge, glioblastoma, microRNA-23b are the three dominant approaches to study the effect of microRNA (miRNA)-induced loss of function in a specific cell type of interest. However, inhibitor oligonucleotides are only effective in short-term (24-72 h) experiments due to their unpredictable degradation. miRNA-knockout animal models are an alternative for functional studies in vivo; however, the time-consuming procedures and costs required to generate genetic knockout animals often preclude the extensive use of the method. The use of an miRNA sponge, which contains multiple target sites that are complementary to an miRNA of interest, is a dominant-negative method. These transcripts can specifically sequester target miRNAs and thereby prevent the miRNAs from binding to their endogenous target mRNAs $(4,5)$. Indeed, the experimental application of miRNA sponge technology has attracted increasing interest for in vitro and in vivo applications, suggesting that this approach can greatly aid in the understanding of miRNA functioning (6-9).

Glioblastoma multiforme (GBM), World Health Organization (WHO) grade IV, is the most common and biologically aggressive malignant glioma. Diffuse infiltration, robust angiogenesis, uncontrolled cellular proliferation, intense resistance to apoptosis and rampant genomic instability are the hallmark features that make this disease incurable (10). miR-23b has been extensively studied in several pathologies and has been found to be upregulated in human GBM regardless of the subtype being studied, compared to adjacent non-neoplastic brain tissues $(11,12)$. However, the expression of miR-23b varies between cancer types, which adds to the complexity of understanding the role of this miRNA in etiopathology $(8,11,13-15)$. It was reported that miR-23b was downregulated in human colon cancer samples and potently mediated multiple steps of metastasis in vivo, including tumor growth, invasion and angiogenesis (8). However, in contrast to colon cancer, GBM showed a relatively high expression level of miR-23b, and our previous study demonstrated that the downregulation of miR-23b suppressed tumor growth by targeting VHL (11). Therefore, we aimed to examine the pivotal role of miR-23b downregulation in tumor angiogenesis, invasion and migration in GBM. Although we previously used chemically modified antisense oligonucleotides to knock down the expression of miR-23b (11), in the present study, we designed an miRNA sponge as a decoy target to effectively 
and specifically inhibit miR-23b. However, many cells both in vitro and in vivo are resistant to the uptake of oligonucleotides. Thus, to mimic the downregulation of miR-23b in GBM, we employed a lentivirus-mediated miR-23b sponge to execute miR-23b downregulation in glioma cells in Petri dishes and in an orthotopic model.

In the present study, we showed that the stable lentivirusmediated introduction of an miR-23b sponge both in vitro and in vivo downregulated miR-23b, resulting in reduced glioma angiogenesis, invasion and migration. These findings facilitate a better understanding of the role of miR-23b in the molecular pathogenesis of cancer and suggest that miR-23b might be a candidate for the treatment of GBM patients.

\section{Materials and methods}

Cells and cell culture. Human U87, LN229 and U251 GBM cells and human umbilical vein endothelial cells (HUVECs) were obtained from the China Academia Sinica Cell Repository (Shanghai, China). The GBM cells were maintained in Dulbecco's modified Eagle's medium (DMEM; Gibco) supplemented with $10 \%$ fetal bovine serum (FBS; HyClone) and incubated at $37^{\circ} \mathrm{C}$ in a $5 \% \mathrm{CO}_{2}$ atmosphere. The HUVECs were grown in endothelial growth medium, EGM-2 (Gibco, EBM-2 supplemented with growth factors and other supplements) with $2 \%$ FBS.

Lentivirus preparation and in vitro infection. Lentiviral vectors expressing a scrambled control, an miR-23b sponge, or luciferase were all generated by GenePharma (Shanghai, China). Cell infections were conducted according to GenePharma's recommendations.

RNA extraction and quantitative real-time analysis. Total RNA was extracted using TRIzol (Invitrogen). Real-time quantification for miRNA measurement was performed with the the Hairpin-it ${ }^{\mathrm{TM}}$ miRNAs qPCR Quantitation kit (GenePharma) and the DNA Engine Opticon 2 Two-Color Real-Time PCR Detection system (Bio-Rad Laboratories). miR-23b expression was normalized to the expression of U6 small nucleolar RNA (snRU6). Each reaction was performed in triplicate. Quantification was performed using the $2^{-\Delta \Delta \mathrm{Ct}}$ method.

Western blot analysis and immunohistochemistry (IHC). Western blotting and IHC assays were performed as previously described (16). The antibodies used were anti-VHL, anti-VEGF, anti-MMP2, anti-CD31, anti-MMP9 and antiHIF-1 $\alpha$ from Santa Cruz Biotechnology; anti-E-cadherin and anti-ZEB1 from Cell Signaling Technology; and anti- $\beta$-catenin from Signalway Antibody. Western blot bands were subjected to densitometric analyses using ImageJ (National Institutes of Health). The mean relative intensities of the relevant protein bands were quantified and normalized to GAPDH levels.

Tubule formation assay. A Matrigel (BD Biosciences) assay was performed to assess endothelial tubule formation in vitro. Ninety-six-well plates were coated with $75 \mu \mathrm{l}$ of Matrigel/well and incubated at $37^{\circ} \mathrm{C}$ for $35 \mathrm{~min}$. HUVECs were harvested and suspended in EBM supplemented with an equal volume of the conditioned medium (CM) from glioma cells treated with the scrambled control or the miR-23b sponge. In each well, $1 \times 10^{4} \mathrm{HUVECs}$ were plated and maintained at $37^{\circ} \mathrm{C}$. The experiments were performed in triplicate, repeated at least once and analyzed in a double blind fashion by two observers.

In vitro invasion assays. Transwell membranes coated with Matrigel (BD Biosciences) were used to quantify glioma cell invasion in vitro. Transfected cells were plated at $2 \times 10^{4} /$ well in the upper chamber in serum-free medium; medium containing $10 \%$ FBS was added to the lower chamber. After an 18-h incubation, the non-invading cells were removed from the top well with a cotton swab and the cells on the bottom were fixed in methanol, stained with $0.1 \%$ crystal violet, and photographed in three independent x10 fields/well. The data represent the mean \pm standard error $(\mathrm{SE})$ of three independent experiments.

In vitro scratch assay. At $72 \mathrm{~h}$ after infection with a scrambled control-expressing or an miR-23b sponge-expressing lentivirus, an artificial wound was created using a $200-\mu 1$ pipette tip, and the cells were further incubated. To analyze cell migration into the wound, images were obtained at 0 and $18 \mathrm{~h}$ using a digital camera system coupled to a microscope. Image J was used to determine the relative migration distance as the reduction of the width of the open area. A statistical analysis of the results of three experiments was performed using the Student's t-test.

Nude mouse tumor intracranial model. Before implantation, U87 cells that were co-infected with a luciferase-expressing lentivirus and an miR-23b sponge-expressing or a scrambled control-expressing lentivirus were serially diluted in a 24-well plate prior to luminescence imaging. The U87 cells were then injected intracranially into 5-week-old BALB/c-nu mice. After 15 days, the tumors were measured by luminescence imaging of whole mice using an IVIS Lumina Imaging System (Xenogen). Paraffin-embedded sections $(8 \mu \mathrm{m})$ were stained with hematoxylin and eosin (H\&E) and used for IHC analysis. When experimental animals were used, the research followed the internationally recognized guidelines on animal welfare and local and national regulations.

Statistical analysis. Statistical analysis was performed using the SPSS Graduate Pack, version 11.0, statistical software (SPSS). Descriptive statistics, including the mean and the SE and a one-way analysis of variance (ANOVA), were used to determine statistically significant differences. The overall survival curves were plotted according to the Kaplan-Meier method, with the log-rank test being applied for comparison. $\mathrm{P}<0.05$ was considered to indicate a statistically significant result.

\section{Results}

Knockdown of miR-23b expression in glioma cells via the lentivirus-mediated miR-23b sponge. In order to knock down miR-23b expression, we constructed an miRNA sponge consisting of a decoy vector expressing tandem repeated miRNA binding sites (Fig. 1A). The binding sites for miR-23b 
A

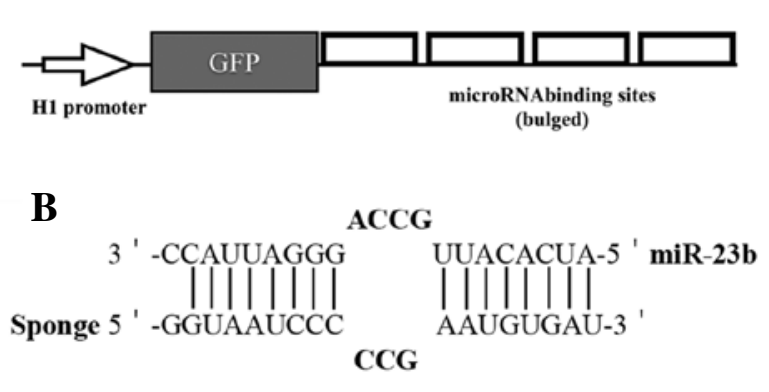

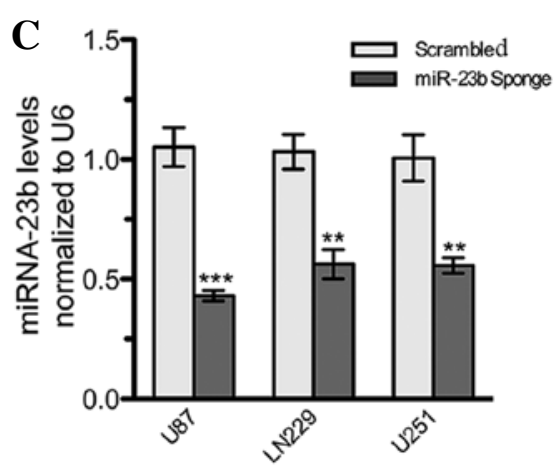

D

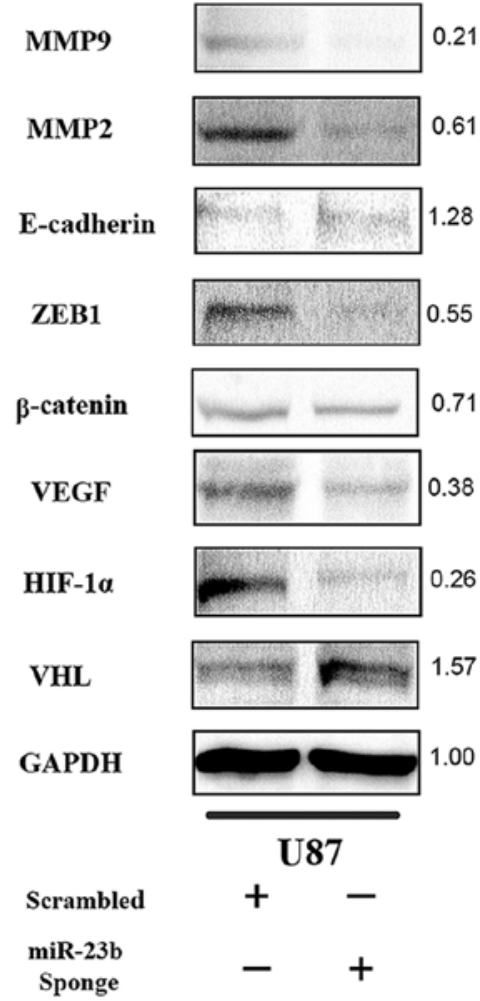

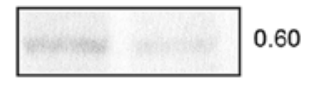
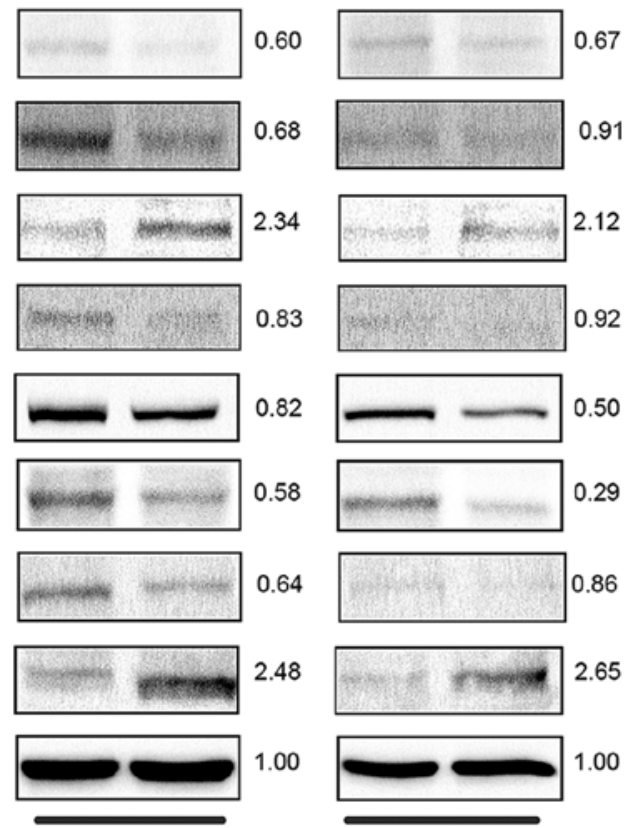

LN229

U251
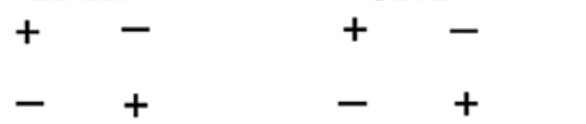

Figure 1. Validation of the miR-23b sponge in three glioma cell lines (U87, LN229 and U251). (A) The miR-23b decoy vector was constructed by inserting four tandem-arrayed microRNA binding sites to downstream of the $\mathrm{H} 1$ promoter. (B) Imperfect pairing between a microRNA and a sponge with a bulged binding sites is illustrated for miR-23b. We designed the sponge with a bulge to protect against endonucleolytic cleavage by Argonaute 2. (C) qRT-PCR quantification of miR-23b expression in glioma cell lines after infection with the scrambled control-expressing or miR-23b sponge-expressing lentivirus. All values are expressed as means \pm SEM. ${ }^{* *} \mathrm{P}<0.01$ and ${ }^{* * *} \mathrm{P}<0.001$. (D) Western blot analysis of the expression of proteins that are associated with tumor angiogenesis, invasion and migration. The numbers indicate the relative protein expression in glioma cell lines infected with the miR-23b sponge-expressing lentivirus as compared to the cells infected with the scrambled control.

were perfectly complementary in the seed region, with a bulge at positions 9-12 to prevent RNA interference-type cleavage and degradation of the sponge RNA (Fig. 1B). As a control, we constructed another vector with repeated scrambled binding sites. Due to the toxicity and low efficiency of plasmid transfection, we utilized a lentivirus as the gene delivery vector, a system that can deliver an miR-23b sponge with relatively low toxicity and high efficiency $(17,18)$. We engineered two lentiviral systems to express the miR-23b sponge or scrambled control and then infected three glioma cell lines (U87, LN229 and U251). To verify the efficacy of endogenous miR-23b inhibition using the lentivirus-mediated miR-23b sponge, we performed qRT-PCR analysis of the glioma cell lines after infection. The results showed that miR-23b expression in all three cell lines was reduced by $\sim 50 \%$ (Fig. 1C).
Downregulation of miR-23b impairs the glioma cell functional capacity for angiogenesis, invasion and migration. To evaluate the functional consequences of expression of the miR-23b sponge in glioma cells, three glioma cell lines (U87, LN229 and U251) were infected with a lentivirus expressing either the miR-23b sponge or scrambled control. We performed western blot analysis to assay the expression of the miRNA target protein (VHL) (19) and proteins associated with tumor angiogenesis [(HIF-1 $\alpha)(19)$ and VEGF (19)], invasion [( $\beta$-catenin) (20), ZEB1 (21) and E-cadherin (20)] and migration [(MMP2 (22) and MMP9 (22)]. As shown in Fig. 1D, the inhibition of miR-23b elevated the expression of tumor-suppressive proteins (VHL and E-cadherin) and abrogated the expression of oncogenic proteins (HIF-1 $\alpha$, VEGF, MMP2, MMP9, $\beta$-catenin and 
A

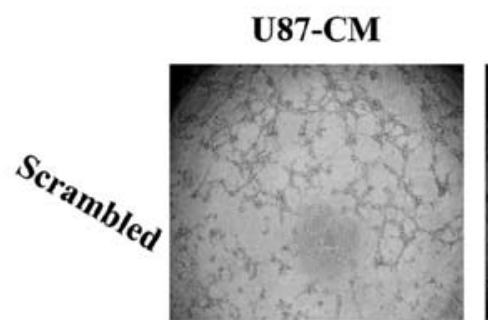

LN229-CM
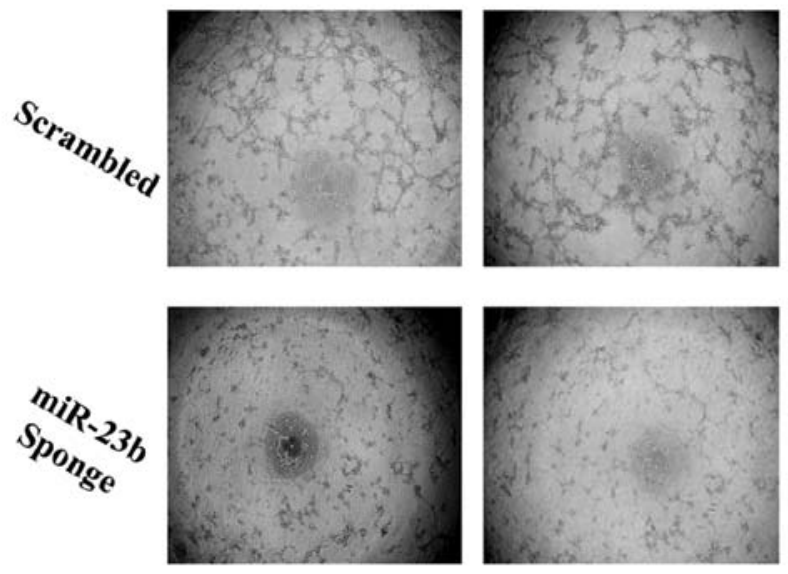

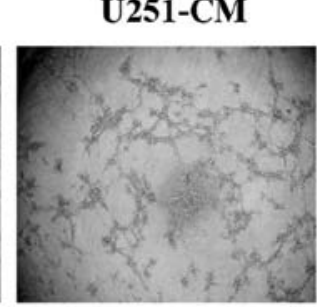

B

U87

LN229

U251
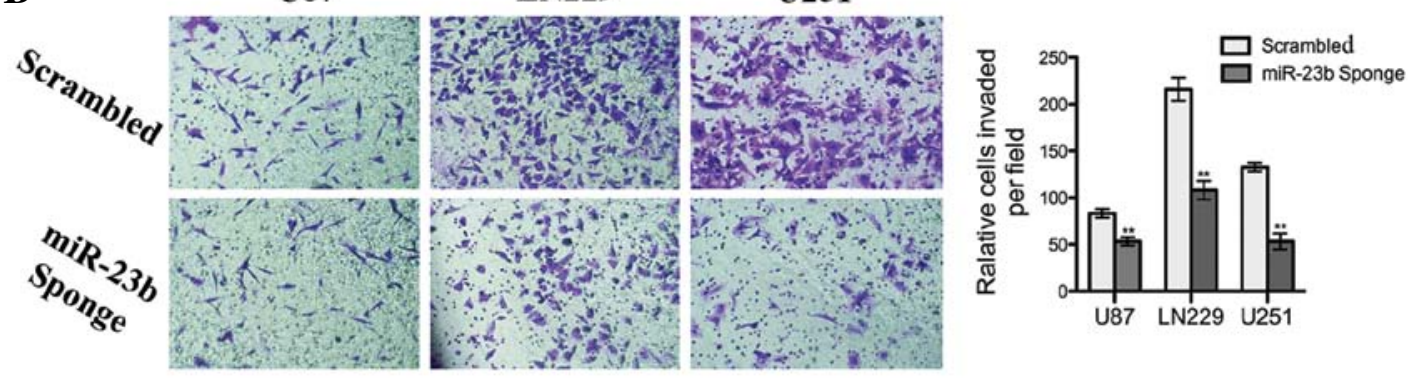

C

U87

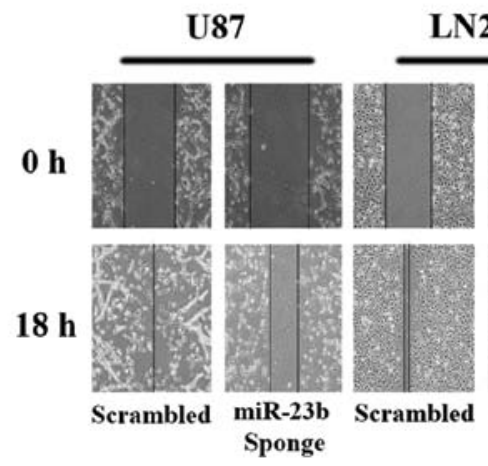

LN229
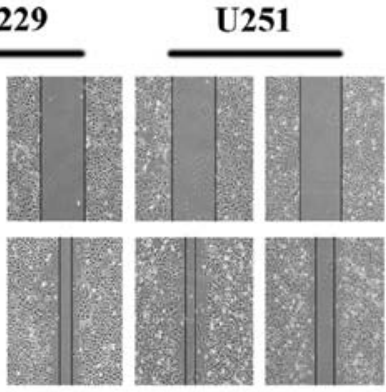

miR-23b
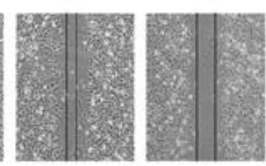

Sponge

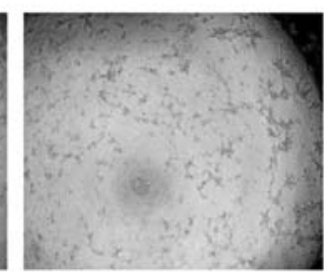


A
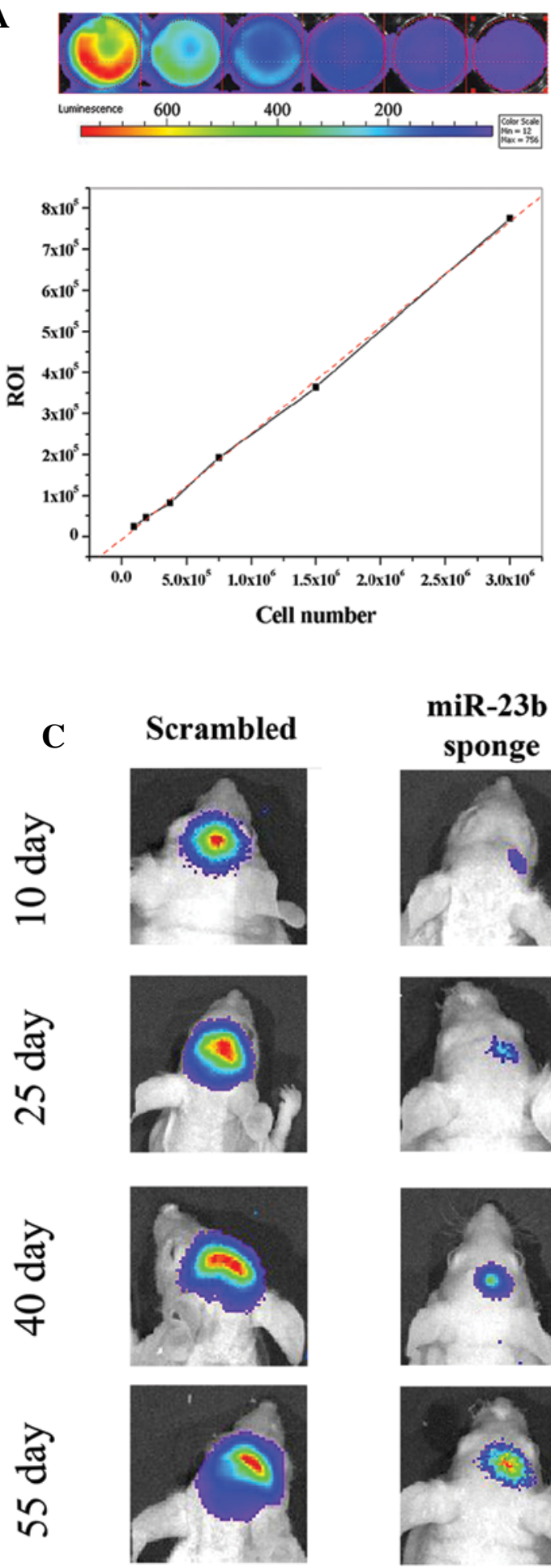

miR-23b
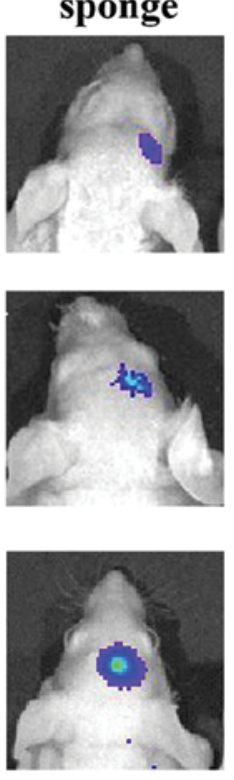

B

Bright

field

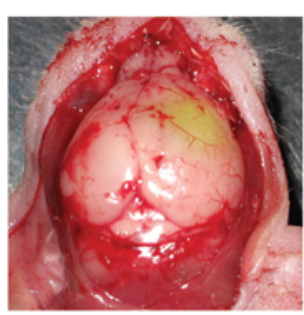

GFP

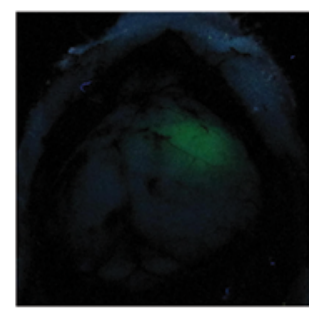

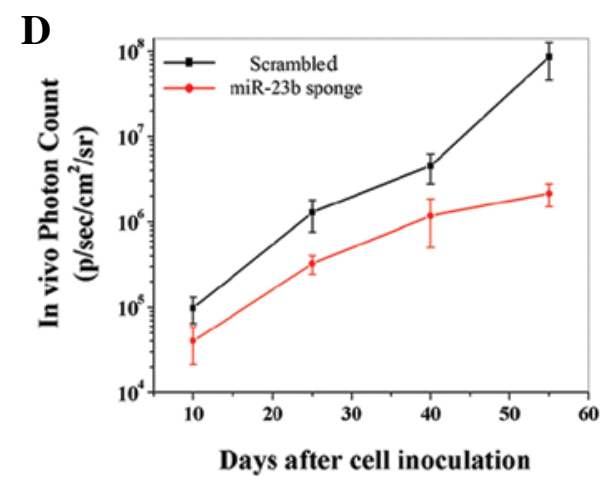

$\mathbf{E}$

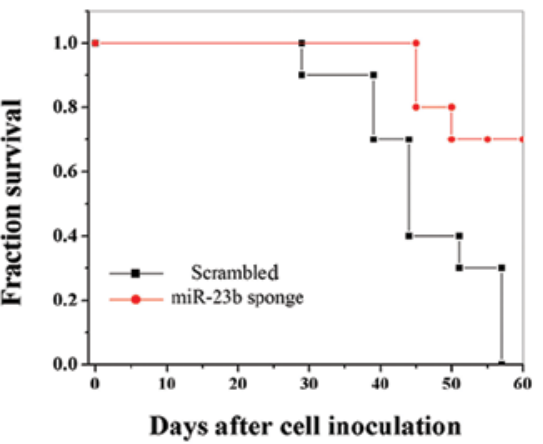

$\begin{array}{cccccccc}\text { Scrambled } & 10 & 10 & 10 & 9 & 7 & 4 & 0 \\ \begin{array}{c}\text { miR-23b } \\ \text { sponge }\end{array} & 10 & 10 & 10 & 10 & 10 & 8 & 7\end{array}$

Figure 3. Introduction of the miR-23b sponge by a lentivirus inhibits U87 tumor growth in vivo. (A) Luminescence analysis after the serial dilution of the implanted U87 cells in a 24-well plate showed that the luminescent signal intensity corresponded to the proportion of live cells within each well. (B) Representative image of a tumor expressing GFP. (C) Representative images of bioluminescence in mice intracranially injected with U87 cells treated with the scrambled control or miR-23b sponge on days 10,25, 40 and 55 after implantation. (D) Quantitative analysis of these images revealed inhibition of growth of the miR-23b sponge-treated U87 tumors in vivo. (E) Animal survival analysis. All of the values are expressed as means \pm SEM.

miR-23b downregulation was found to inhibit angiogenesis, invasion, and migration in vitro, we predicted the inhibitio of cell growth in an orthotopic graft after treatment with the miR-23b sponge. Prior to implantation, we conducted a serial-dilution validation using U87 cells infected with the luciferase-expressing lentivirus (Fig. 3A). An excellent linear 
A
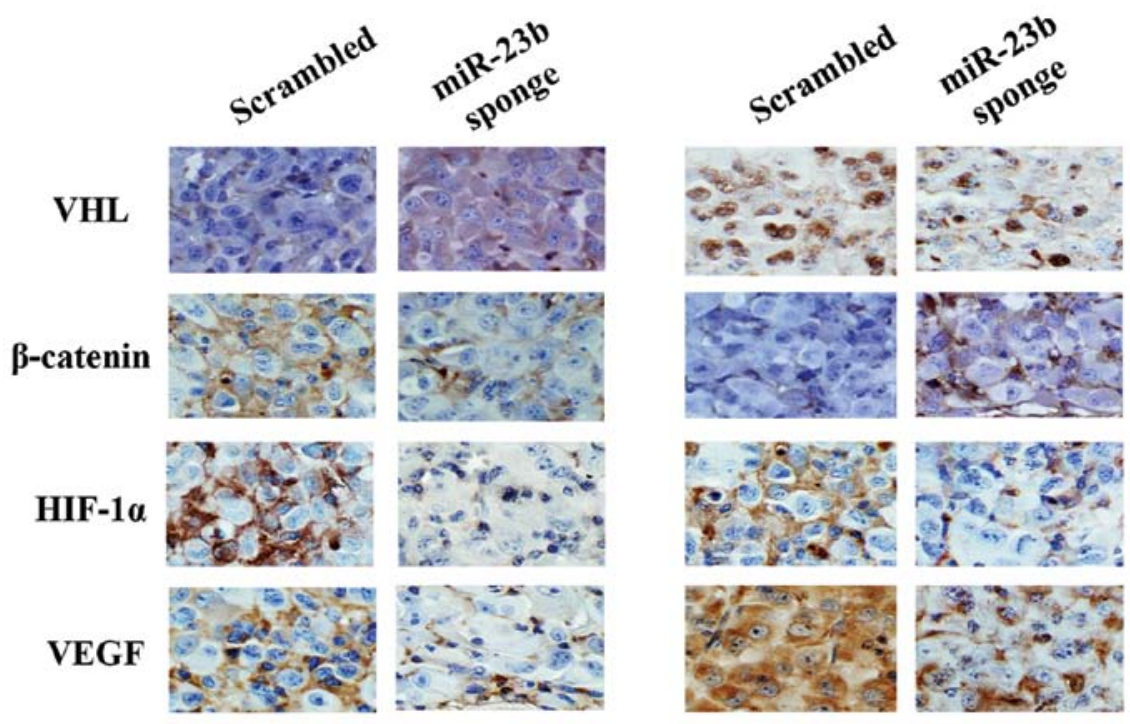

ZEB1

E-caherin

B

Scrambled

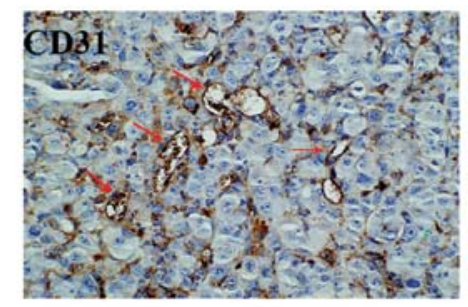

C

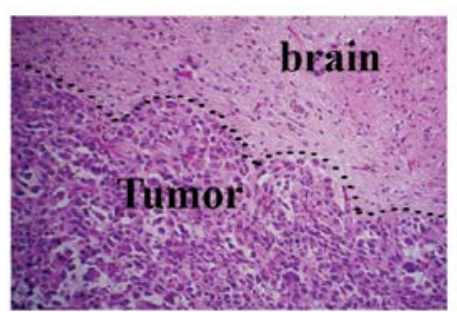

miR-23b sponge

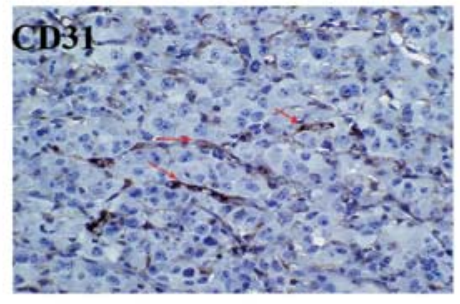

miR-23b sponge

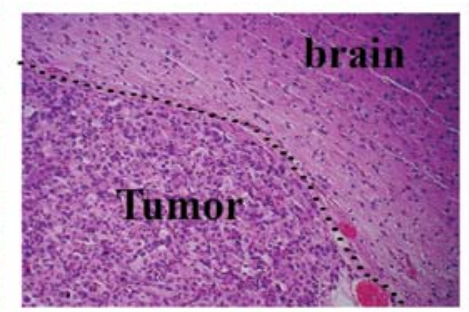

Figure 4. miR-23b downregulation affects U87 cell functional capacities for angiogenesis, invasion and migration in vivo. (A) IHC analysis of the expression of proteins that are associated with tumor angiogenesis, invasion and migration. (B) CD31-positive vessels (red arrow) showed a significant decrease in vessel number. (C) Histological comparison of the tumors derived from the transplantation of U87 cells treated with the scrambled control or miR-23b sponge. miR23b-downregulated tumors displayed less peripheral invasion of the surrounding brain, as evidenced by single cells and cell clusters.

correlation was observed between the number of live cells and luciferase activity. Fig. 3B shows a visualization of a U87 tumor expressing GFP in situ. In vivo imaging analysis of the mice every 15 days for 45 days revealed the suppressed growth rate of the miR-23b sponge-treated U87 cells (Fig. 3C and D). Furthermore, treatment with the miR-23b sponge was associated with a significantly longer survival rate of the mice (Fig. 3E).

miR-23b sponge-treated U87 cells have reduced angiogenic, invasive and migratory potential in vivo. Consistent with our data from the in vitro assays, an IHC analysis showed decreased expression of HIF- $1 \alpha$, $\beta$-catenin, MMP2, MMP9, VEGF and ZEB1 and increased expression of VHL and E-cadherin after treatment with the mR-23b sponge (Fig. 4A). To further investigate angiogenesis within the U87 orthotopic graft, we stained tumor sections with an anti-CD31 antibody, which demonstrated a reduction in CD31-positive vessels after miR-23b sponge treatment, further supporting the anti-angiogenic effect of miR-23b inhibition in vivo (Fig. 4B). H\&E staining of the miR-23b-downregulated tumors revealed less peripheral invasion, which may have resulted from reduced invasive and migratory abilities (Fig. 4C). In summary, our results demonstrate anti-angiogenic, anti-invasive and anti-migratory implications of the introduction of the miR-23b sponge.

\section{Discussion}

As there is increasing evidence that miRNAs participate in carcinogenesis, the identification of physiologically relevant and/or therapeutically promising miRNAs has become an essential task $(6,23,24)$. Our previous study showed that 
miR-23b is overexpressed in glioma samples and cell lines (11). However, miR-23b has been found to be downregulated in human colon cancer samples and to potently mediate the multiple steps of metastasis in vivo, including tumor growth, invasion and angiogenesis (8). However, there has been no systematic report to date on the effects of miR-23b on GBM pathological processes $(11,25)$. Therefore, we investigated the role of miR-23b in glioma angiogenesis, invasion and migration, characteristics that make GBM incurable.

As miR-23b is overexpressed in gliomas, we first knocked down miR-23b expression in glioma cell lines. There are three general methods for miRNA loss-of-function studies: genetic knockout, antisense oligonucleotide inhibitors (1-3) and sponges (4). Although these oligonucleotide-based methods have been successfully applied, the techniques do elicit off-target side-effects and unwanted toxicity. In contrast, specific miRNA sponges are superior for substantially inhibiting target miRNAs, monitoring the transfection efficacy and specifically selecting a particular subpopulation of transfected cells $(4,5)$. We, therefore, replaced antisense oligonucleotides with an miR-23b sponge, which was engineered in a lentiviral vector to allow more effective gene delivery. In the present study, the lentiviral vector encoding the miR-23b sponge was found to sufficiently silence miR-23b activity, resulting in the detection of broad changes in the angiogenesis, invasion and migration of glioma cell lines (U87, L229 and U251). After replacing antisense nucleotide with the miR-23b sponge, we performed western blot analysis to detect alterations in the expression of proteins associated with angiogenesis, invasion and migration. In our previous study (11), we demonstrated that miR-23b directly targeted VHL and consequently inhibited the $\beta$-catenin/Tcf-4 and HIF-1 $\alpha /$ VEGF signaling pathways (11). HIF-1 $\alpha$ and VEGF are well-established pro-angiogenic proteins among cell lines and are negatively regulated by VHL (26). ZEB1 and E-cadherin are known for their implications in epithelial-mesenchymal transition (EMT), which stabilizes the invasive mesenchymal phenotype of epithelial tumor cells, and $\beta$-catenin is involved by interacting closely with E-cadherin (27). Matrix metalloproteinases (MMPs) are a family of enzymes responsible for the proteolytic processing of extracellular matrix structural proteins, which regulate endothelial cell migration (22). Therefore, the reduced expression of HIF- $1 \alpha$, VEGF, ZEB1, $\beta$-catenin and MMP2/9 and the elevation of VHL and E-cadherin levels indicated that miR-23b inhibition exerted an anticancer effect on all of the glioma cell lines tested. Consistent with the western blotting results, the suppression of angiogenic, invasive, and migratory capabilities was confirmed in glioma cell lines by tubule formation, Transwell and scratch assays, respectively. We speculated that similar tumor inhibition would be observed in vivo, and experiments using an orthotopic glioma mouse model affirmed this assumption. Importantly, the miR-23b spongetreated mice demonstrated a more favorable outcome when compared to the mice treated with the scrambled control. We proposed that the impairment of angiogenesis, invasion, and migration that decreased the growth of the orthotopic graft was responsible for this difference in survival. Consistent with the in vitro results, histological staining demonstrated a reduction in vessels and less peripheral invasion in the miR-23b sponge-treated orthotopic grafts.
Based on these observations, miR-23b-based anticancer therapy (such as an miR-23b sponge) is promising and can be developed, either alone or in combination, with current targeted therapies, with the goal of improving disease responses and increasing cure rates. This therapy may be particularly essential for GBM, which has a uniformly poor prognosis due to diffuse infiltration and robust angiogenesis. However, tissuespecific delivery, potential off-target effects, and biological safety must be addressed before miRNA-based therapeutics can be adopted.

\section{Acknowledgements}

The present study was supported by the National High Technology Research and Development Program 863 (2012AA02A508), the China National Natural Scientific Foundation (81001128, 81172406, 81372703), the Research Fund for the Doctoral Program of Higher Education of China (20111202110004), the Natural Science Foundation of Tianjin Municipal Science and Technology Commission (12JCZDJC24300) and the Tianjin City High School Science \& Technology Fund (2009CD01, 20090228).

\section{References}

1. Krützfeldt J, Rajewsky N, Braich R, et al: Silencing of microRNAs in vivo with 'antagomirs'. Nature 438: 685-689, 2005.

2. Meister G, Landthaler M, Dorsett Y and Tuschl T: Sequencespecific inhibition of microRNA- and siRNA-induced RNA silencing. RNA 3: 544-550, 2004.

3. Ørom UA, Kauppinen S and Lund AH: LNA-modified oligonucleotides mediate specific inhibition of microRNA function. Gene 372: 137-141, 2006.

4. Ebert MS, Neilson JR and Sharp PA: MicroRNA sponges: competitive inhibitors of small RNAs in mammalian cells. Nat Methods 9: 721-726, 2007.

5. Ebert MS and Sharp PA: MicroRNA sponges: progress and possibilities. RNA 16: 2043-2050, 2010.

6. Valastyan S, Reinhardt F, Benaich N, et al: A pleiotropically acting microRNA, miR-31, inhibits breast cancer metastasis. Cell 137: 1032-1046, 2009.

7. Lin J, Teo S, Lam DH, Jeyaseelan K and Wang S: MicroRNA-10b pleiotropically regulates invasion, angiogenicity and apoptosis of tumor cells resembling mesenchymal subtype of glioblastoma multiforme. Cell Death Dis 3: e398, 2012.

8. Zhang H, Hao Y, Yang J, et al: Genome-wide functional screening of miR-23b as a pleiotropic modulator suppressing cancer metastasis. Nat Commun 2: 554, 2011.

9. Xu W, Liu M, Peng X, et al: miR-24-3p and miR-27a-3p promote cell proliferation in glioma cells via cooperative regulation of MXI1. Int J Oncol 42: 757-766, 2013.

10. Furnari FB, Fenton T, Bachoo RM, et al: Malignant astrocytic glioma: genetics, biology, and paths to treatment. Genes Dev 21: 2683-2710, 2007.

11. Chen L, Han L, Zhang K, et al: VHL regulates the effects of miR-23b on glioma survival and invasion via suppression of HIF-1/VEGF and $\beta$-catenin/Tcf-4 signaling. Neuro Oncol 14: 1026-1036, 2012.

12. Ciafrè SA, Galardi S, Mangiola A, et al: Extensive modulation of a set of microRNAs in primary glioblastoma. Biochem Biophys Res Commun 334: 1351-1358, 2005.

13. Wang KC, Garmire LX, Young A, et al: Role of microRNA-23b in flow-regulation of $\mathrm{Rb}$ phosphorylation and endothelial cell growth. Proc Natl Acad Sci USA 107: 3234-3239, 2010.

14. Jin L, Wessely O, Marcusson EG, Ivan C, Calin GA and Alahari SK: Prooncogenic factors miR-23b and miR-27b are regulated by Her2/Neu, EGF, and TNF- $\alpha$ in breast cancer. Cancer Res 73: 2884-2896, 2013.

15. Rogler CE, LeVoci L, Ader T, et al: MicroRNA-23b cluster microRNAs regulate transforming growth factor-beta/bone morphogenetic protein signaling and liver stem cell differentiation by targeting Smads. Hepatology 50: 575-584, 2009. 
16. Zhou X, Ren Y, Moore L, et al: Downregulation of miR-21 inhibits EGFR pathway and suppresses the growth of human glioblastoma cells independent of PTEN status. Lab Invest 90 144-155, 2010.

17. Rubinson DA, Dillon CP, Kwiatkowski AV, et al: A lentivirusbased system to functionally silence genes in primary mammalian cells, stem cells and transgenic mice by RNA interference. Nat Genet 33: 401-406, 2003

18. Dull T, Zufferey R, Kelly M, et al: A third-generation lentivirus vector with a conditional packaging system. J Virol 72: 8463-8471, 1998.

19. Hutson TE, Davis ID, Machiels JH, et al: Biomarker analysis and final efficacy and safety results of a phase II renal cell carcinoma trial with pazopanib (GW786034), a multi-kinase angiogenesis inhibitor. J Clin Oncol 158: 5046, 2008.

20. Lu Z, Ghosh S, Wang Z and Hunter T: Downregulation of caveolin-1 function by EGF leads to the loss of E-cadherin, increased transcriptional activity of $\beta$-catenin, and enhanced tumor cell invasion. Cancer Cell 4: 499-515, 2003.

21. Burk U, Schubert J, Wellner U, et al: A reciprocal repression between ZEB1 and members of the miR-200 family promotes EMT and invasion in cancer cells. EMBO Rep 9: 582-589, 2008.
22. Wang L, Zhang ZG, Zhang RL, et al: Matrix metalloproteinase 2 (MMP2) and MMP9 secreted by erythropoietin-activated endothelial cells promote neural progenitor cell migration. J Neurosci 26: 5996-6003, 2006.

23. Kota J, Chivukula RR, O'Donnell KA, et al: Therapeutic microRNA delivery suppresses tumorigenesis in a murine liver cancer model. Cell 137: 1005-1017, 2009.

24. Zhang J, Han L, Ge Y, et al: miR-221/222 promote malignant progression of glioma through activation of the Akt pathway. Int J Oncol 36: 913-920, 2010.

25. Geng J, Luo $\mathrm{H}, \mathrm{Pu}$ Y, et al: Methylation mediated silencing of miR-23b expression and its role in glioma stem cells. Neurosci Lett 528: 185-189, 2012

26. Foxler DE, Bridge KS, James V, et al: The LIMD1 protein bridges an association between the prolyl hydroxylases and VHL to repress HIF-1 activity. Nat Cell Biol 14: 201-208, 2012.

27. Schmalhofer O, Brabletz $S$ and Brabletz T: E-cadherin, $\beta$-catenin, and ZEB1 in malignant progression of cancer. Cancer Metastasis Rev 28: 151-166, 2009. 